\title{
Adaptive CNN-based Private LTE Solution for Fair Coexistence with Wi-Fi in Unlicensed Spectrum
}

\author{
Merkebu Girmay, Vasilis Maglogiannis, Dries Naudts, Jaron Fontaine, Adnan Shahid, Eli De Poorter \\ and Ingrid Moerman
}

\begin{abstract}
Department of Information Technology (INTEC), Ghent University, Ghent, Belgium
\{merkebutekaw.girmay, vasilis.maglogiannis, dries.naudts, jarone.fontaine, adnan.shahid, eli.depoorter, ingrid.moerman\}

@ugent.be
\end{abstract}

\begin{abstract}
Recently, the expansion of wireless network deployments is resulting in increased scarcity of available licensed radio spectrum. As the domain of wireless communications is progressing rapidly, many industries are looking into wireless network solutions that can increase their productivity. Private LTE is a promising wireless network solution as it can be customised independently without the control of a mobile network operator while providing reliable and spectrum efficient services. For this reason, the deployment of Private LTE in the unlicensed spectrum and its coexistence with $\mathrm{Wi}-\mathrm{Fi}$ is becoming a popular topic in research. In this paper, we propose a coexistence scheme for private LTE network in unlicensed spectrum that enables a fair spectrum sharing with co-located Wi-Fi networks. This is achieved by exploiting various LTE frame configurations consisting of different combinations of downlink, uplink, special subframe and muted subframes. The configuration of a single frame is decided based on a rule based algorithm that exploits Wi-Fi spectrum occupancy statistics that is obtained from a technology recognition system which is based on a Convolutional Neural Network. The performance of the proposed private LTE scheme and its coexistence with Wi-Fi is investigated for different traffic scenarios showcasing how the proposed scheme can lead to a harmless coexistence of LTE and Wi-Fi.
\end{abstract}

Index Terms-Private adaptive LTE, Wi-Fi, CNN, Technology Recognition, AI/ML, Fair Coexistence.

\section{INTRODUCTION}

The advances in various wireless communications has led to an exponential growth in wireless transmitted traffic. This, combined with limited availability of licensed spectrum for cellular networks, has inspired the use of unlicensed bands for LTE [1]. Although, the use of LTE in unlicensed bands enhances its capacity, it raises serious concerns about its coexistence with other co-located networks operating in the same band.

$\mathrm{Wi}-\mathrm{Fi}$ is one of the common technologies that operate in the unlicensed spectrum and it utilizes the carrier sense multiple access with collision avoidance (CSMA/CA) protocol to coexist with various other wireless technologies in the unlicensed spectrum, whereas traditional LTE uses continuous signal transmission with limited time gaps even in the absence of data traffic. Considering these operating characteristics in both systems, Wi-Fi seems to have very limited opportunities to use the channel compared to an LTE under a coexistence scenario, resulting in a performance degradation for the $\mathrm{Wi}$ Fi. Hence, several techniques have been proposed aiming to achieve harmonious coexistence between LTE and other wellestablished technologies in the unlicensed spectrum [2].

LTE Unlicensed (LTE-U) is the first technology that uses the $5 \mathrm{GHz}$ unlicensed band for opportunistic offload of mobile network traffic and it is standardized by LTE-U Forum. LTE Licensed Assisted Access (LAA) is the standard version of the unlicensed LTE developed by the 3GPP. LTE-U uses a mechanism called Carrier-Sensing Adaptive Transmission which is based on LTE duty-cycling its transmissions. On the other hand, LAA uses a mechanism called Listen Before Talk (LBT). LBT uses Clear Channel Assessment (CCA) before any transmission in unlicensed spectrum. CCA uses energy detection to determine transmit opportunities [3].

In LTE-U and LTE-LAA, an operator who owns a licensed frequency band will opportunistically offload LTE traffic in the unlicensed spectrum. Hence, both technologies are not suitable for enterprise owners, service providers, operators etc. who intend to operate exclusively in the unlicensed spectrum. Private LTE is a technology proposed to decouple LTE from the operators and enable the LTE operation solely in the unlicensed spectrum. The main purpose of a private LTE network is to connect devices belonging to an enterprise (normally across a campus or site), and to totally secure the data by avoiding transmitting it through the core network of a mobile operator. Full private ownership of the whole LTE network, including base stations and core, has several advantages such as high availability, simpler traffic priority management and easier upgrade and maintenance [4].

Private LTE technology solutions include MulteFire technology and Citizens Broadband Radio service (CBRS). MulteFire builds on elements of LTE LAA and combines the high performance of LTE with the simple deployment of Wi-Fi. Despite its performance advantages, Multefire has standardization differences from legacy LTE. The modifications on physical layer channels, synchronization, reference signals, random access procedure, paging and mobility management make it incompatible with the legacy LTE User Equipment (UE) [5]. On the other hand, CBRS has a similar architecture to legacy LTE but it requires a Spectrum Allocation System (SAS) that controls and assigns spectrum and an Environmental Sensing capability (ESC) component which senses the band for incumbent transmissions [6]. 
In this paper, we have analyzed the coexistence of a private LTE that solely operates in unlicensed spectrum and Wi-Fi with real hardware-based implementation. Our private LTE is implemented mainly based on legacy LTE which is compatible with commercial off-the-shelf (COTS) LTE UE and the performance of the coexisting Private LTE and Wi-Fi is evaluated. We analyze how the two technologies can fairly coexist in real time based on a rule based frame configuration selection algorithm that exploits Wi-Fi spectrum occupancy statistics obtained from the technology recognition. The key contributions of this work include:

- Proposal of a flexible and adaptive LTE scheme for harmless coexistence with Wi-Fi.

- Autonomous selection of the optimal configuration parameters that offer fair coexistence.

- Support Frequency Division Duplex (FDD) and Time Division Duplex (TDD) mode to use unlicensed band for both uplink (UL) and downlink (DL) traffic.

- Frequency agnostic solution that supports both private and unlicensed bands.

- Compliance with COTS LTE UE, as far as the desired frequency is supported by the radio frequency front-end.

- Compliance with existing 3GPP standards, since the modifications are limited to the LTE scheduler, ensuring flexible mapping of ACK/NACK transmission.

The remainder of this paper is organized as follows. Section II reviews some recent related studies on the coexistence of LTE and Wi-Fi. Section III discusses the proposed LTE coexistence mechanism. Section IV describes the equipment used and the hardware implementation setup. Section V explains the results obtained in different experimental scenarios and provides a detailed performance evaluation of the proposed coexistence mechanism. Finally, Section VI concludes the article and discusses plans for future work.

\section{RELATED WORK}

Recently, extensive research has been conducted to assure fair coexistence between LTE and Wi-Fi networks in the unlicensed spectrum. In [7], we performed our first study that investigates the impact of a traditional LTE network operating in unlicensed spectrum on Wi-Fi by using COTS equipment. The obtained results show that the performance of Wi-Fi can be significantly affected by LTE. The results clearly indicate that coexistence mechanisms are required in order to enable fair and harmonious spectrum sharing between LTE and other co-located technologies such as Wi-Fi.

Taking the impact of LTE transmissions on Wi-Fi into account, 3GPP announced the LTE LAA standards in Release 13 [8], including the description of a CCA procedure. 3GPP Release 13 allows LTE LAA operation in the $5 \mathrm{GHz}$ band for DL traffic only. In 3GPP Release 14 [9], the standard has been extended to allow both DL and UL traffic in the $2.4 \mathrm{GHz}$ unlicensed band. The Carrier Aggregation mechanism that is used for the transmission in the unlicensed spectrum via a secondary cell operating alongside the primary cell has been introduced in 3GPP LTE Release 10 [10].
Based on the 3GPP specifications and spectrum regulations of different regions different coexistence mechanisms between Wi-Fi and LTE in unlicensed spectrum are proposed in [11][12]. In these papers, a duty-cycle mechanism that selects the suitable probability to access the channel and transmission duration is used. This duty-cycle mechanism is implemented by introducing muted subframes in LTE which helps to ensure proportional fairness among LTE and Wi-Fi [13]. During muted subframes, the coexisting LTE refrains from transmitting and as a result $\mathrm{Wi}-\mathrm{Fi}$ gets more opportunities to access the channel. In [14], it is shown that the throughput of a colocated Wi-Fi network is affected by the sequence of muted subframes.

The previously mentioned studies mainly consider a DL LTE to coexist with Wi-Fi where supportive licensed spectrum is used for control signals and/or UL transmissions. In [14][15], coexistence of LTE in TDD mode with Wi-Fi is studied. These studies also examine the effect of using muted subframe on the coexistence performance of LTE and Wi-Fi. However, these papers only make simulation based evaluations. Furthermore, the existing works do not consider selection of adaptive frame configuration based on Wi-Fi traffic statistics.

In our previous work [16], a detailed review of Artificial Intelligence (AI)/Machine Learning (ML) based coexistence studies was performed and a Convolutional Neural Network (CNN) that identifies real time over-the-air LTE and Wi-Fi transmissions was developed. Several statistics of co-located technologies are used in order to select the appropriate transmission and muting period of an adaptive LTE scheme.

According to the best of our knowledge, the current literature lacks of LTE adaptive frame configuration selection mechanisms that can offer harmless coexistence between LTE and Wi-Fi with real hardware implementation. This paper presents a hardware implementation and performance evaluation of an adaptive LTE frame configuration selection based LTE and Wi-Fi coexistence. In this paper, the CNN technology implemented in [16] is used for technology recognition by private LTE eNodeB (eNB) to select an appropriate frame configuration which is the combination of muted subframe, special subframe (SS), UL and DL subframes in a single frame. The channel occupancy of Wi-Fi is detected by the technology recognition system and it is used to make a rule based selection among a set of coexisting LTE frame configurations. Furthermore, we consider a flexible mapping of LTE subframe ACK/NACK transmission based on the selected frame configuration.

\section{Proposed Private LTE coexistence Scheme}

This section describes the proposed private LTE coexistence scheme. In our previous work [16], a technology recognition based LTE and Wi-Fi coexistence scheme is developed which enables eNB to transmit in the unlicensed spectrum, while the UL traffic is transmitted via the primary licensed band. This work extends the coexistence scheme so that both DL and UL transmissions are set to coexist in the unlicensed spectrum. The developed solution supports both TDD and FDD mode. 
The remainder of this paper focuses on TDD mode since it does not require a paired spectrum for UL and DL traffic. As a result, TDD is more suitable for private LTE deployments and especially in an unlicensed spectrum since both the DL and UL transmissions take place in a single channel.

In our previous work [7], we have shown that the performance of Wi-Fi is highly affected by LTE transmissions even if LTE transmits only control signals. In this work, different LTE frame configurations are proposed to achieve coexistence with Wi-Fi. Table I shows the 8 different subframe configurations with different number of muted subframes which are used by the proposed coexistence scheme. In this table the DL, UL, muted and SS are represented by D, U, B and S respectively. The muted LTE subframes are used in order to enhance the coexistence of LTE with Wi-Fi. In this work, a muted subframe is a subframe where both the UE and eNB remain silent so that co-located networks can get transmission opportunities. In case of subframe index $0,1,5$ and 6 a muted subframe holds synchronization signals only to maintain the synchronization between the eNB and the UE. The synchronization signals are transmitted periodically every $5 \mathrm{~ms}$ and they have very short duration (one OFDM symbol period). Hence, potential interference with Wi-Fi can be considered negligible. The muted subframes are set to be consecutive to achieve better coexistence performance [12]. Table I also shows the muted percentage of the spectrum in a single LTE frame.

TABLE I: Proposed Coexistence Frame Configurations

\begin{tabular}{|c|c|c|c|c|c|c|c|c|c|c|c|}
\hline Frame & \multicolumn{10}{|c|}{ subframe Index } & \multirow{2}{*}{$\underset{\%}{\text { Muted }}$} \\
\hline Conf. & 0 & 1 & 2 & 3 & 4 & 5 & 6 & 7 & 8 & 9 & \\
\hline $\mathrm{C}_{0}$ & $\mathrm{D}$ & $\mathrm{S}$ & $\mathrm{U}$ & $\mathrm{U}$ & $\mathrm{U}$ & $\mathrm{D}$ & $\mathrm{D}$ & D & $\mathrm{D}$ & $\mathrm{D}$ & $7.14 \%$ \\
\hline $\mathrm{C}_{1}$ & D & $\mathrm{S}$ & $\mathrm{U}$ & U & $\mathrm{U}$ & B & D & D & D & D & $17.14 \%$ \\
\hline $\mathrm{C}_{2}$ & $\mathrm{D}$ & $S$ & $\mathrm{U}$ & $\mathrm{U}$ & $\mathrm{B}$ & $\mathrm{B}$ & $\mathrm{D}$ & $\mathrm{D}$ & $\mathrm{D}$ & $\mathrm{D}$ & $27.14 \%$ \\
\hline $\mathrm{C}_{3}$ & $\mathrm{D}$ & $S$ & $\mathrm{U}$ & $\mathrm{U}$ & $\mathrm{B}$ & $\mathrm{B}$ & $\mathrm{B}$ & $\mathrm{D}$ & $\mathrm{D}$ & $\mathrm{D}$ & $37.14 \%$ \\
\hline $\mathrm{C}_{4}$ & $\mathrm{D}$ & $\mathrm{S}$ & $\mathrm{U}$ & B & B & B & B & $\mathrm{D}$ & $\mathrm{D}$ & $\mathrm{D}$ & $47.14 \%$ \\
\hline $\mathrm{C}_{5}$ & $\mathrm{D}$ & $\mathrm{S}$ & $\mathrm{U}$ & B & B & B & B & B & $\mathrm{D}$ & $\mathrm{D}$ & $57.14 \%$ \\
\hline $\mathrm{C}_{6}$ & $\mathrm{D}$ & $\mathrm{S}$ & $\mathrm{U}$ & B & B & B & B & B & B & $\mathrm{D}$ & $67.14 \%$ \\
\hline $\mathrm{C}_{7}$ & D & $\mathrm{S}$ & $\mathrm{U}$ & B & B & B & B & B & B & B & $77.14 \%$ \\
\hline
\end{tabular}

In the proposed coexistence scheme, the configuration of the $\mathrm{SS}$ is also selected to enhance coexistence with Wi-Fi. 3GPP has defined 9 different configurations for the SS [9] . Each configuration consists of DL Pilot Time Slot (DwPTS), Guard Period (GP) and UL Pilot Time Slot (UpPTS) periods, which consist of variable number of OFDM symbols. The GP is a transition gap between the DL and UL. In this work, the ' 0 ' SS configuration is selected, as it offers the longest GP. This way, a potential co-located Wi-Fi network earns transmission opportunities before the coexistence scheme adapts. According to this SS configuration, 3, 10 and 1 LTE OFDM symbols are allocated for the DwPTS, GP and UpPTS respectively.

In this work, the ACK/NACK time delays of UL and DL LTE data are also modified for a better LTE and WiFi coexistence. LTE transmits user traffic in the DL and UL using Physical Downlink Shared Channel (PDSCH) and Physical Shared Uplink Channel (PUSCH), respectively. In addition to user traffic, several resource blocks are allocated for control traffic such as synchronization signals, reference signals, hybrid automatic repeat request (HARQ) ACK/NACK signals, etc. In Frequency Division Duplex (FDD) mode, a UE completes the decoding of the PDSCH and transmits HARQ ACK or NACK after 4ms. But in TDD, a UE cannot transmit the response of every transmission interval (TTI) in such a fixed timing as in FDD. The HARQ ACK or NACK transmission must wait until the next UL subframe according to the UL/DL configuration. Thus, the ACK/NACK response of each PDSCH is transmitted $\mathrm{K}$ milliseconds after it is received by the UE. Similarly, the eNB transmits ACK/NACK for the UL subframes L milliseconds after it is received.

Considering the resource blocks of physical UL control channel (PUCCH) and the TDD UL/DL configurations, 3GPP provides time delay mapping of HARQ ACK/NACK for every DL and UL subframe [9]. Table II shows this timing configurations. For every subframe, the first set of numbers given in brackets are values of $\mathrm{K}$ that correspond to ACK/NACK delay of received PDSCH by the UE. Similarly, the second set of numbers indicate values of $\mathrm{L}$ that correspond to ACK/NACK delay of received PUSCH by the eNB. In this work, we consider the TDD UL/DL configuration 3 as initial reference based on which the coexistence scheme is implemented, as it provides a good proportion between DL and UL subframes during an LTE frame. Hence, regarding the HARQ ACK/NACK time mapping of TDD configuration 3, at subframe 2 the numbers $(7,6,11)(6)$ show that the values of $\mathrm{K}$ are 7,6 and 11, while the value of $\mathrm{L}$ is 6 . This indicates that at subframe 2, the UE transmits the ACK/NACK of the PDSCH that was received before $7 \mathrm{~ms}$, $6 \mathrm{~ms}$, and $11 \mathrm{~ms}$ respectively. Similarly, the ACK/NACK of a PUSCH that was received before $6 \mathrm{~ms}$ is transmitted by the eNB at subframe 2 .

Table III shows the proposed $\mathrm{K}$ and $\mathrm{L}$ delay mapping of DL and UL ACK/NACK respectively for each frame configuration shown in Table I. Likewise Table II, the first set of numbers given within brackets are values of $\mathrm{K}$ and the second set of numbers are the values of $\mathrm{L}$. The ACK/NACK delay values $(\mathrm{K}, \mathrm{L})$ are selected so that no ACK/NACK signal is transmitted during SS and muted subframe, aiming to minimize the interference with Wi-Fi.

The proposed private LTE eNB selects an appropriate frame configuration among the ones listed in Table I based on statistics obtained from the technology recognition system. The CNN used in this technology recognition is trained and validated using commercial hardware for both the LTE and Wi-Fi networks and the In-phase and Quadrature-phase (I/Q) samples are collected from a Universal Software Radio Peripheral (USRP) at center frequency of $2.437 \mathrm{GHz}$. The maximum channel occupancy of Wi-Fi for each proposed LTE frame configuration was experimentally measured and a rule based frame configuration selection algorithm was developed based on that. The maximum channel occupancy of Wi-Fi was measured by transmitting iPerf Wi-Fi traffic along with concurrent LTE iPerf traffic transmission for each proposed LTE frame configuration. The maximum Wi-Fi channel occupancy values obtained by the technology recognition system are $3 \%$, 
TABLE II: HARQ ACK/NACK subframe association index (K),(L)

\begin{tabular}{|c|c|c|c|c|c|c|c|c|c|c|}
\hline \multirow{2}{*}{$\begin{array}{l}\text { TDD } \\
\text { Conf }\end{array}$} & \multicolumn{10}{|c|}{ Subframe Index } \\
\hline & 0 & 1 & 2 & 3 & 4 & 5 & 6 & 7 & 8 & 9 \\
\hline 0 & $(-),(-)$ & $(-),(-)$ & $(6),(4)$ & $(-),(7)$ & $(4),(-)$ & $(6),(-)$ & $(-),(-)$ & $(6),(-)$ & $(4),(7)$ & $(4),(6)$ \\
\hline 1 & $(-),(-)$ & $(-),(-)$ & $(7,6),(4)$ & $(4),(6)$ & $(-),(-)$ & $(-),(-)$ & $(-),(-)$ & $(7,6),(4)$ & $(4),(6)$ & $(-),(-)$ \\
\hline 2 & $(-),(-)$ & $(-),(-)$ & $(8,7,4,6),(6)$ & $(-),(-)$ & $(-),(-)$ & $(-),(-)$ & $(-),(-)$ & $(8,7,4,6),(6)$ & $(-),(-)$ & $(-),(-)$ \\
\hline 3 & $(-),(-)$ & $(-),(-)$ & $(7,6,11),(6)$ & $(6,5),(6)$ & $(5,4),(6)$ & $(-),(-)$ & $(-),(-)$ & $(-),(-)$ & $(-),(-)$ & $(-),(-)$ \\
\hline 4 & $(-),(-)$ & $(-),(-)$ & $(12,8,7,11),(6)$ & $(6,5,4,7),(6)$ & $(-),(-)$ & $(-),(-)$ & $(-),(-)$ & $(-),(-)$ & $(-),(-)$ & $(-),(-)$ \\
\hline 5 & $(-),(-)$ & $(-),(-)$ & $(13,12,9,8,7,4,11,6),(6)$ & $(-),(-)$ & $(-),(-)$ & $(-),(-)$ & $(-),(-)$ & $(-),(-)$ & $(-),(-)$ & $(-),(-)$ \\
\hline 6 & $(-),(-)$ & $(-),(-)$ & (7),(4) & $(7),(6)$ & $(5),(6)$ & $(-),(-)$ & $(-),(-)$ & (7),(4) & (7),(7) & $(-),(-)$ \\
\hline
\end{tabular}

$12 \%, 24 \%, 35 \%, 42 \%, 52 \%, 60 \%$ and $68 \%$ for $\mathrm{C}_{0}, \mathrm{C}_{1}, \mathrm{C}_{2}$, $\mathrm{C}_{3}, \mathrm{C}_{4}, \mathrm{C}_{5}, \mathrm{C}_{6}$ and $\mathrm{C}_{7}$ frame configurations respectively.

Based on the proposed rule-based coexistence scheme, the private LTE selects the frame configuration $\mathrm{C}_{0}$ as long as WiFi is not detected. The eNB checks the channel occupancy of Wi-Fi to determine whether there is Wi-Fi traffic. Once Wi-Fi traffic is detected, Algorithm 1 is used. As it can be observed in this algorithm, the eNB switches to monitoring mode using configuration $\mathrm{C}_{7}$ for configurable amount of time (Monitoring $\left.{ }_{\text {Period }}\right)$. In this mode, the eNB transmits only at subframe 0 and 2, while it remains silent for the rest of the frame, giving long channel access opportunities to Wi-Fi. During the monitoring mode, the eNB monitors the channel occupancy statistics reported by the technology recognition and based on these statistics, it selects a frame configuration that can provide the required channel occupancy of Wi-Fi. The eNB uses this configuration for configurable amount of time (Hold $\mathrm{Heriod}_{\text {) }}$ until it switches to monitoring mode again in order to re-evaluate the requirements of the Wi-Fi traffic. This way, the LTE frame configurations are continuously updated to match the Wi-Fi traffic demands.

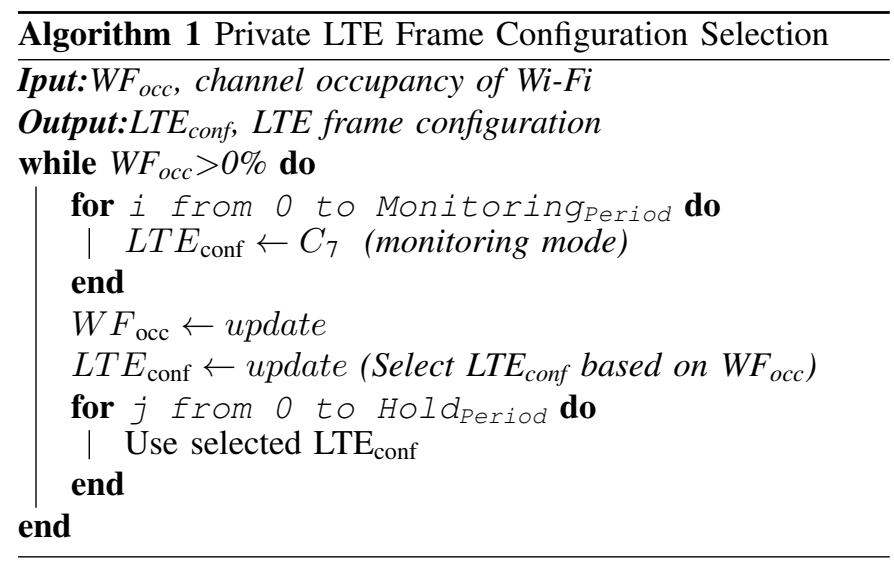

Another challenge that must be addressed during introduction of muted subframe is the noise estimation. In LTE DL, every OFDM frame has cell-specific reference symbols, which facilitate the noise power estimation. In the proposed frame configurations, it is described that muted subframes can only hold synchronization signals (i.e. reference symbols and other control signals are not transmited). Therefore, reference signals are not used to estimate noise of the channel at the receiver side. Hence, in this work, the noise estimation is done based on the synchronization signals of LTE [17].

\section{EXPERIMENTATION TOPOLOGY AND USED EQUIPMENT}

This section describes the equipment and the topology used for the experiments performed during this study. Fig. 1 shows the used experiment topology. The figure shows Wi-Fi access point and station, LTE eNB and UE and a server where the technology recognition solution runs. In this work, the average throughput of LTE and Wi-Fi in each considered scenario was obtained by measuring TCP iPerf throughput for 30s duration with an interval of 1s. The technology recognition runs in in a separate server which has USRP board connected to it for fetching the I/Q samples transmitted by different co-located technologies over-the-air. The outputs of the technology recognition computation are conveyed to eNB host PC using ZeroMQ [18].

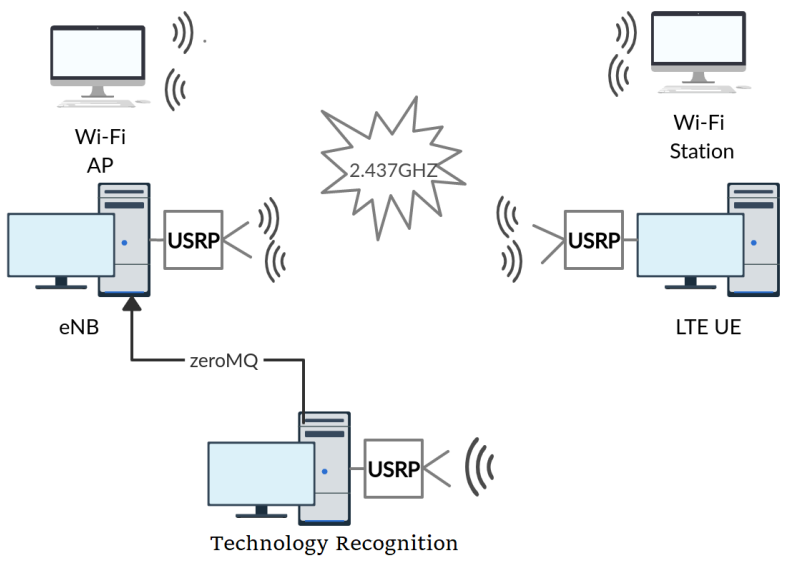

Fig. 1: Experiment Topology

The LTE network has been deployed based on srsLTE open source code [19] and software-defined radio (SDR) platforms. srsLTE is a highly modular LTE software framework developed by SRS and includes complete SDR LTE applications for the eNB, the UE and the Evolved Packet Core (EPC) side. The srsLTE framework is LTE Release 10 compliant and FDD mode is implemented in both eNB and UE side, while TDD Mode option is also included in the UE side. The radio part of the LTE network uses USRP B210 boards as its SDR platform. The LTE software runs on top of Gigabyte BRIX Compact PCs and the USRP boards are connected to the PC as a front end. 
TABLE III: Modified HARQ ACK/NACK subframe association index (K),(L)

\begin{tabular}{|c|c|c|c|c|c|c|c|c|c|c|}
\hline \multirow{2}{*}{$\begin{array}{c}\text { Coexistence } \\
\text { conf }\end{array}$} & \multicolumn{9}{|c|}{ Subframe Index } \\
\cline { 2 - 11 } & $\mathbf{0}$ & $\mathbf{I}$ & $\mathbf{2}$ & $\mathbf{3}$ & $\mathbf{4}$ & $\mathbf{5}$ & $\mathbf{6}$ & $\mathbf{7}$ & $\mathbf{8}$ & $\mathbf{9}$ \\
\hline $\mathrm{C}_{0}$ & $(-),(-)$ & $(-),(-)$ & $(7,6,11),(6)$ & $(6,5),(6)$ & $(5,4),(6)$ & $(-),(-)$ & $(-),(-)$ & $(-),(-)$ & $(-),(-)$ & $(-),(-)$ \\
\hline $\mathrm{C}_{1}$ & $(-),(-)$ & $(-),(-)$ & $(6,11),(6)$ & $(6,5),(6)$ & $(5,4),(6)$ & $(-),(-)$ & $(-),(-)$ & $(-),(-)$ & $(-),(-)$ & $(-),(-)$ \\
\hline $\mathrm{C}_{2}$ & $(-),(-)$ & $(-),(-)$ & $(12,11),(6)$ & $(6,5,4),(6)$ & $(-),(-)$ & $(-),(-)$ & $(-),(-)$ & $(-),(-)$ & $(-),(-)$ & $(-),(-)$ \\
\hline $\mathrm{C}_{3}$ & $(-),(-)$ & $(-),(-)$ & $(12,11,5),(6)$ & $(4,5),(7)$ & $(-),(-)$ & $(-),(-)$ & $(-),(-)$ & $(-),(-)$ & $(-),(-)$ & $(-),(-)$ \\
\hline $\mathrm{C}_{4}$ & $(-),(-)$ & $(-),(-)$ & $(13,12,11,5,4),(6)$ & $(-),(-)$ & $(-),(-)$ & $(-),(-)$ & $(-),(-)$ & $(-),(-)$ & $(-),(-)$ & $(-),(-)$ \\
\hline $\mathrm{C}_{5}$ & $(-),(-)$ & $(-),(-)$ & $(13,12,11,4),(6)$ & $(-),(-)$ & $(-),(-)$ & $(-),(-)$ & $(-),(-)$ & $(-),(-)$ & $(-),(-)$ & $(-),(-)$ \\
\hline $\mathrm{C}_{6}$ & $(-),(-)$ & $(-),(-)$ & $(13,12,11),(7)$ & $(-),(-)$ & $(-),(-)$ & $(-),(-)$ & $(-),(-)$ & $(-),(-)$ & $(-),(-)$ & $(-),(-)$ \\
\hline $\mathrm{C}_{7}$ & $(-),(-)$ & $(-),(-)$ & $(12,11),(8)$ & $(-),(-)$ & $(-),(-)$ & $(-),(-)$ & $(-),(-)$ & $(-),(-)$ & $(-),(-)$ & $(-),(-)$ \\
\hline
\end{tabular}

For the purpose of this study, the eNB part of the srsLTE software suite has been extended to support the frame configurations listed in Table I. The eNB is also modified to use Algorithm 1 to select a frame configuration based on the Wi-Fi channel occupancy report obtained from the technology recognition. The eNB and UE are configured to operate at WiFi channel 6 of the $2.4 \mathrm{GHz}$ band with a center frequency of $2.437 \mathrm{GHz}$ and $10 \mathrm{MHz}$ bandwidth. The srsLTE UE is also configured to use a PSS Zadoff-Chu sequence-based noise estimation. Similarly, a Wi-Fi network that operates in the same frequency as the LTE is set at a close range. The Wi-Fi network is configured in an infrastructure mode and consists of two Zotac nodes. One node operates as an access point while the other operates as a station. All the Wi-Fi nodes use a Qualcomm Atheros AR928X wireless network adapter together with a ath9k driver. The Wi-Fi network is set to operate in a $802.11 \mathrm{~g}$ mode.

\section{EXPERIMENTATION RESULTS}

In this section, the results obtained during different scenarios of the experimental study are presented. Initially, the Wi-Fi performance was tested without any interference from the LTE and an average throughput of $27.8 \mathrm{Mbps}$ is obtained. Similarly, when the LTE operates using a $\mathrm{C}_{0}$ frame configuration, it obtains an average throughput of $18.06 \mathrm{Mbps}$ and $8.82 \mathrm{Mbps}$ for DL and UL respectively. In this initial performance evaluation of the LTE, the Wi-Fi transmission was turned off to avoid interference.

Fig. 2a shows the time vs power measurement of the LTE traffic for a single LTE frame. This specific measurement is captured for a single LTE frame, when the eNB uses a $\mathrm{C}_{4}$ frame configuration. The measurement is obtained by transmitting DL and UL data traffic simultaneously. The graph shows the DL, UL, SS and muted subframes in a single frame. From the figure it can be observed that, the muted frames are totally off so that they can be used for Wi-Fi transmissions. In Fig.2b, the time vs power signal measurement of simultaneous traffic by LTE and Wi-Fi with a duration of $10 \mathrm{~ms}$ is shown. As it can be observed from the figure, the Wi-Fi traffic exploits the muted subframes for accessing the channel.

In Fig. 3, the spectrogram of the LTE and Wi-Fi signals presents the signal strength of both technologies for $20 \mathrm{~ms}$ period in a $20 \mathrm{MHz}$ bandwidth. The yellow, short, solid rectangular shapes show the signal strength of the LTE subframes, whereas the yellow, long, vertical, dashed lines on the spectrogram represent the strength of the Wi-Fi signal. Fig. 3a shows the spectrogram of concurrent LTE and Wi-FI traffic at $\mathrm{C}_{0}$ frame configuration. When the Wi-Fi traffic is detected, the eNB switches to monitoring mode, selecting the $\mathrm{C}_{7}$ frame configuration in order to evaluate the Wi-Fi traffic demand, as shown in Fig.3b. Then, the channel occupancy statistics derived from the technology recognition during the monitoring phase are used to select an appropriate frame configuration. As shown in Fig. 3c, the eNB selects to use the frame configuration $\mathrm{C}_{5}$ for this specific measurement. The Hold $\mathrm{Period}_{\text {}}$ and monitoring Period $_{\text {used in this specific measurement are } 5}$ and 1 LTE frames respectively.

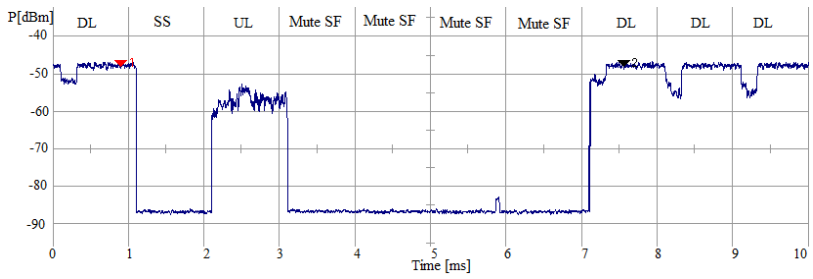

(a)

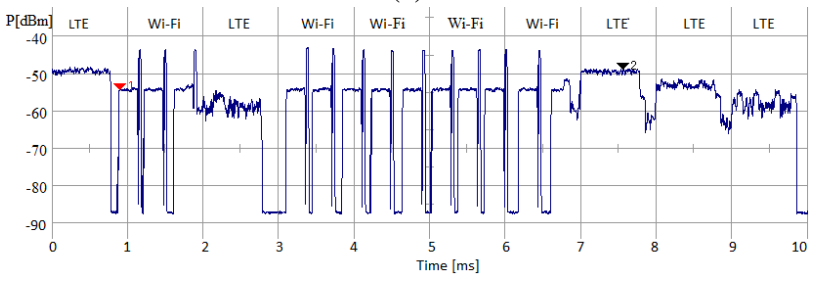

(b)

Fig. 2: Power vs. time graph of a frame a) LTE transmission of $\mathrm{C}_{4}$ configuration b) Concurrent LTE and Wi-Fi Transmission

Figure 4 shows the average throughput of LTE and WiFi at different frame configurations. It can be observed that the throughput of LTE proportionally decreases as frame configurations with more muted subframes are used. On the other hand, the throughput of Wi-Fi rises as the number of muted subframes in the LTE frame increases. The figure shows that the throughput of LTE DL and Wi-Fi are 17.82 Mbps and $1.92 \mathrm{Mbps}$ respectively at LTE frame configuration $\mathrm{C}_{0}$. It can also be observed that the average throughput achieved for LTE DL traffic falls to $3.19 \mathrm{Mbps}$ while the throughput of $\mathrm{Wi}-\mathrm{Fi}$ rises to $16.67 \mathrm{Mbps}$ during the monitoring phase. 


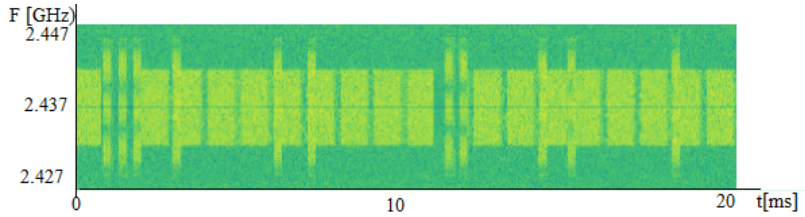

(a)

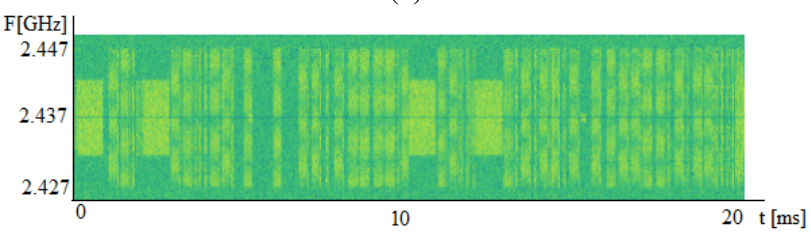

(b)

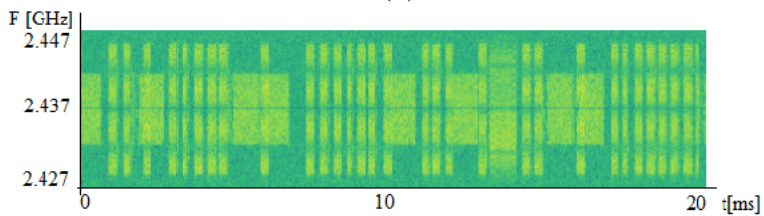

(c)

Fig. 3: Spectrogram of concurrent LTE and Wi-Fi traffic for frame configuration a) $\mathrm{C}_{0}$ b) $\mathrm{C}_{7}$ c) $\mathrm{C}_{5}$

As the results show, the experimentation results show that the CNN based technology recognition can be used to detect the Wi-Fi traffic characteristics. The derived statistics can be exploited by the proposed adaptive LTE scheme to enable harmonious coexistence under continuously changing traffic.

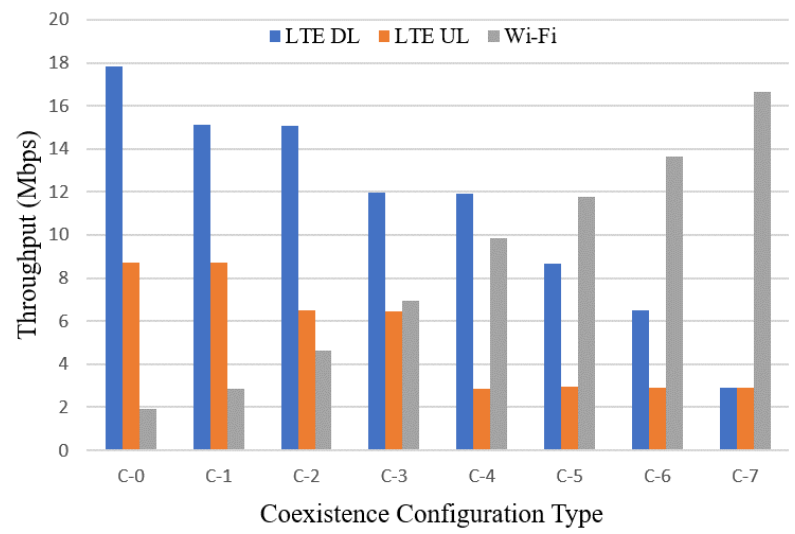

Fig. 4: Throughput of Concurrent LTE and Wi-Fi Traffic

\section{CONCLUSion AND FutURe WORKS}

This study has shown how a private LTE can coexist with a Wi-Fi traffic in unlicensed spectrum. The experimental results show that a private LTE can exploit muted subframes to coexist with a co-located Wi-Fi. It is also shown that different configurations of DL, UL and muted subframes in an LTE frame can be used for different Wi-Fi traffic demand scenarios. In this work, technology recognition statistics reports are used to monitor the traffic demand of a co-located Wi-Fi.
In this paper, a rule based algorithm is used to select an LTE frame configuration as a proof of concept aiming to show how a flexible and adaptive LTE can coexist with a Wi-Fi by exploiting a wireless environment statistics reported in realtime by AI/ML. In this study, LTE adapts according to the Wi-Fi requirements. However, it is very important that the LTE performance is guaranteed as well. Towards this direction, in the near future, a more robust frame configuration selection mechanism based on ML will be developed. Such mechanism can take into account several important statistics that are required for a harmless coexistence such as, monitoring period configuration, Wi-Fi inter-packet interval and packet length, LTE and Wi-Fi transmitting queue lengths, channel occupancy of LTE, overlapping transmissions and other Quality of Service requirements to enhance the capability of the system to respond to a rapidly changing traffic.

\section{REFERENCES}

[1] Signals Research Group, "The prospect LTE Wi-Fi sharing unlicensed spectrum," Qualcomm, San Diego, CA, USA, White Paper, Feb. 2015.

[2] C. Cano and D. J. Leith, "Coexistence of WiFi and LTE in unlicensed bands: A proportional fair allocation scheme," in Proc. IEEE Int. Conf. Commun. Workshop (ICCW), Jun. 2015, pp. 2288-2293.

[3] L. Falconetti et al., "Design and evaluation of licensed assisted access LTE in unlicensed spectrum," IEEE Wireless Commun., vol. 23, no. 6, dec. 2016, pp. 24-30.

[4] Gabriel Brown, "Private LTE networks," Qualcomm, white paper, July 2017.

[5] MulteFire Alliance, Available from: http://www.multefire.org/

[6] CBRSA-TS-1001-V1.0.0, "CBRS Network Services Requirements", October 2017.

[7] V. Maglogiannis, D. Naudts, P. Willemen, and I. Moerman, "Impact of LTE operating in unlicensed spectrum on Wi-Fi using real equipment," in Proc. IEEE Global Telecommun. Conf. (GLOBECOM). Washington, DC, USA, Dec. 2016, pp. 1-9.

[8] 'Evolved Universal Terrestrial Radio Access (E-UTRA); Physical Layer Procedures," Release 13, 3GPP TS, New Orleans, LA, USA, Sep. 2016.

[9] "Evolved Universal Terrestrial Radio Access (E-UTRA); Physical Layer Procedures," Release 14, 3GPP TS, Sapporo, Japan, Sep. 2017

[10] "Evolved Universal Terrestrial Radio Access (EUTRA); Carrier Aggregation; Base Station (BS) radio transmission and reception," Release 10, 3GPP TR, 2011.

[11] Baoan Jia and Meixia Tao, "A Channel Sensing Based Design for LTE in Unlicensed Bands,' IEEE International Conference on Communications Workshop (ICCW), June 2015.

[12] Fabiano S. Chaves, et al., "LTE UL Power Control for the Improvement of LTE/WiFi Coexistence," IEEE Vehicular Technology Conference, 2013, pp. 1-6.

[13] Bocanegra, C.; Kennouche, T.E.; Li, Z.; Favalli, L.; Felice, M.D.; Chowdhury, K. E-Fi: "Evasive Wi-Fi Measures for Surviving LTE within 5 GHz Unlicensed Band. IEEE Trans” Mob. Comput. 2019, 18, 830-844.

[14] M. Abinader, L. Almeida, S. Chaves, M. Cavalcante, D. Vieira, D. Paiva, M. Sobrinho, S. Choudhury, E. Tuomaala, K. Doppler, A. Sousa, "Enabling the Coexistence of LTE and Wi-Fi in Unlicensed Bands," IEEE Comm., 2014, pp. 54-61.

[15] Rojeena Bajracharya, Rakesh Shrestha, Sung Won Kim, "Q-Learning Based Fair and Efficient Coexistence of LTE in Unlicensed Band," Sensors 19 (13): 2875, 2019.

[16] V. Maglogiannis, D. Naudts, A. Shahid, and I. Moerman, "Enhancing the Coexistence of LTE and Wi-Fi in Unlicensed Spectrum Through Convolutional Neural Networks," IEEE Access 2019, 6, pp. 28464-28477.

[17] T. Agrawal, Y. Kumar and S. Ramanath, "Improved noise estimation using synchronization sequences in LTE downlink," 2018 10th International Conference on Communication Systems Networks (COMSNETS), Bengaluru, 2018, pp. 454-456.

[18] Libzmq framework, Available from: https://github.com/zeromq/libzmq

[19] srsLTE software suite, Available from: https:/github.com/srsLTE/srsLTE 